\title{
Ruxolitinib before allogeneic hematopoietic transplantation in patients with myelofibrosis on behalf SFGM-TC and FIM groups
}

\author{
Marie Robin $\mathbb{1}^{1} \cdot$ Raphael Porcher $\mathbb{D}^{2,3} \cdot$ Corentin Orvain $^{4} \cdot$ Jacques-Olivier Bay ${ }^{5}$. Fiorenza Barraco ${ }^{6}$ Anne Huynh ${ }^{7}$. \\ Amandine Charbonnier ${ }^{8} \cdot$ Edouard Forcade $^{9}{ }^{9} \cdot$ Sylvain Chantepie $^{10} \cdot$ Claude Bulabois $^{11}$. \\ Ibrahim Yakoub-Agha ${ }^{12}$ - Marie Detrait ${ }^{13} \cdot$ David Michonneau (D) $^{14} \cdot$ Pascal Turlure $^{15} \cdot$ Nicole Raus $^{16}$. \\ Françoise Boyer $^{4}$ - Felipe Suarez ${ }^{17}$. Laure Vincent ${ }^{18}{ }^{18}$. Stéphanie N. Guyen ${ }^{19}$ • Jérôme Cornillon ${ }^{20}$. \\ Alban Villate ${ }^{21} \cdot$ Brigitte Dupriez $^{22} \cdot$ Bruno Cassinat $^{23} \cdot$ Valérie Rolland $^{24} \cdot$ Marie Hélène Schlageter $^{23}$. \\ Gérard Socié ${ }^{10}{ }^{14}$ Jean-Jacques Kiladjian ${ }^{25}$
}

Received: 4 December 2020 / Revised: 28 January 2021 / Accepted: 19 February 2021 / Published online: 25 March 2021

(c) The Author(s), under exclusive licence to Springer Nature Limited 2021

\begin{abstract}
This multicenter prospective phase 2 trial analyzed disease-free survival (DFS) in myelofibrosis patients receiving ruxolitinib for 6 months before transplantation. Seventy-six patients were recruited. Age-adjusted dynamic international prognostic scoring system was intermediate-1, intermediate-2, and high in 27 (36\%), 31 (41\%), and 18 (24\%) patients. All patients received ruxolitinib from inclusion to conditioning regimen (fludarabine-melphalan) or to progression. A donor was found in 64 patients: 18 HLA-matched sibling donor (MSD), 32 HLA-matched unrelated (UD10/10), and 14 HLA mismatched unrelated donor (UD9/10. Among 64 patients with a donor, $20(31 \%)$ achieved a partial response before transplantation and $59(92 \%)$ could be transplanted after ruxolitinib therapy (18/18 MSD, 30/21 UD10/10, 11/34 UD9/10), of whom 19 (32\%) were splenectomized. Overall survival from inclusion was $68 \%$ at 12 months. One-year DFS after transplantation was 55\%: $83 \%, 40 \%$, and $34 \%$ after MSD, UD10/10 or UD9/10, respectively. Cumulative incidence of grade 2-4 acute graft-versushost disease (GVHD) was $66 \%$ and non-relapse-mortality was $42 \%$ at 12 months. Short course of ruxolitinib before transplantation is followed by a high rate of transplantation. With the platform used in this protocol, outcome was much better in patients transplanted with HLA-matched sibling donor as compared to unrelated donor.
\end{abstract}

\section{Introduction}

Myelofibrosis (MF) is a chronic malignancy, characterized by marrow fibrosis and myeloproliferation due to constitutive activation in the janus-activated kinase (JAK)/signal transducers and activators of transcription signaling pathway, mostly related to mutations in the driver genes $J A K 2$, myeloproliferative leukemia virus (MPL) or

These authors contributed equally: Gérard Socié, Jean-Jacques Kiladjian

Supplementary information The online version contains supplementary material available at https://doi.org/10.1038/s41409021-01252-7.

Marie Robin

marie.robin@aphp.fr

Extended author information available on the last page of the article calreticulin $(C A L R)$. In addition, comprehensive genomic characterization classified accurately this disease [1]. Allogeneic hematopoietic stem cell transplantation (HSCT) is a curative treatment that can be offered to younger, higherrisk patients who are fit enough to undergo this intensive therapy [2]. Unfortunately, there is a substantial proportion of failure after HSCT, either due to disease recurrence, graft rejection, graft-versus-host disease (GVHD), or toxicity. The general health of the patient before HSCT, disease features, and parameters related to the procedure is associated with post-transplant outcome [3-11]. Ruxolitinib, a JAK1/2 inhibitor that reduces inflammation-related general symptoms and splenomegaly, has been approved in many countries for patients with myelofibrosis. Ruxolitinib does not suppress fibrosis, or the malignant clone burden, but, although still a matter of debate, may improve life expectancy in very high-risk patients $[12,13]$. We report here the results of a phase 2 prospective trial in myelofibrosis, using ruxolitinib in treatment-naive patients before HSCT. The 
primary aim was disease-free survival (DFS) 12 months after HSCT. The hypothesis was a reduction of general symptoms and an improvement of performance status before HSCT, which may improve post-HSCT outcome, with a better engraftment, a lower toxicity, and lower mortality. Furthermore, need for splenectomy before HSCT, performed in $40 \%$ of patients in France and potentially complicated by thrombosis or hemorrhage, may be reduced after ruxolitinib [6]. Concerns were that pre-transplant ruxolitinib may be associated with (1) a "withdrawal effect" after stopping ruxolitinib before HSCT, (2) a poor engraftment, (3) an increased risk of infection, and (4) a potential decreased graft-versus-leukemia effect due to its immunosuppressive properties.

\section{Patients and method}

\section{Protocol and patients}

JAK ALLO was a phase-2 trial testing ruxolitinib before HSCT in myelofibrosis and was registered at ClinicalTrials. gov with identifier: NCT01795677. The study was approved by the national legal body and by ethical committee Paris IV, and was conducted in accordance with the Declaration of Helsinki and Good Clinical Practice guidelines, informed consent were obtained for all patients. The primary aim was DFS 1 year after allogeneic transplantation. Secondary objectives were splenectomy rate, engraftment, GVHD incidence, overall survival (OS), NRM, cytokine profile, assessment of quality of life, and symptoms related to the myelofibrosis. Patients with intermediate or high-risk MF according to Lille score [14] or DIPSS [15], naive of JAK inhibitor treatment, aged from 18 to 69 years could be included. Ruxolitinib treatment and search for a donor were started, with the aim of performing the HSCT within 6 months after inclusion. Exclusion criteria, ruxolitinib treatment management, and transplant procedure protocols are detailed in Supplemental Data. Conditioning regimen consisted of fludarabine $30 \mathrm{mg} / \mathrm{m}^{2} /$ day for 3 days and Melphalan $140 \mathrm{mg} / \mathrm{m}^{2}$ for 1 day. GVHD prophylaxis consisted of cyclosporine and mycophenolate mofetil. Anti-thymocyte globulin in the setting of an unrelated donor was left to the physician's discretion. Due to severe adverse events (SAE) and the possible relationship with ruxolitinib or RWS, several amendments had to be made during the protocol, as well as an interruption of inclusions from June 3, 2013 to October 8, 2014 before being validated by legal authorities (Supplemental Data and Fig. S1 for recruitment). Among changes, ruxolitinib discontinuation before transplantation was progressive within 15 days in the initial protocol and abrupt just before melphalan after amendment.

\section{Definitions}

Complete response (CR), partial remission (PR), and spleen response were defined as previously published [16]. CR definition after HSCT was adapted as follows: no constitutional symptoms due to the disease, normal spleen size, normal blood cell count and blood smear, normal marrow biopsy or complete donor chimerism. Marrow biopsy was done at 1-year post transplantation while chimerism was done regularly from day 30 . DFS was defined as survival in CR. Engraftment after transplantation was defined as neutrophil recovery, defined as three consecutive days with a neutrophil count $>0.5 \times 10^{9} / \mathrm{L}$, platelets maintained $>20 \times$ $10^{9} / \mathrm{L}$ without transfusion for more than 7 days. Primary graft failure was defined as the failure to achieve adequate neutrophil and platelet counts (primary) at day 60, secondary graft failure was defined as a decline in counts after achievement of sustained adequate counts (secondary) with loss of graft (recipient chimerism). Acute, hyperacute GVHD, and chronic GVHD were defined according to previously published criteria [17-19].

Ruxolitinib withdrawal syndrome (RWS) was defined according to previous reports $[20,21]$ and is detailed in Supplemental data.

\section{Statistics}

A number of 53 transplanted patients was computed to demonstrate an increase in DFS 12 months after HSCT from 50 to $70 \%$ with a one-sided $5 \%$ significance level and 90\% power [22]. The probability to find a donor in French patients was assumed to be about $70 \%$, which lead to the planning of inclusion for 76 to 80 patients in the study. The statistical analysis was performed in "intent to transplant" considering patients with or without donor at 6 months, and the type of donor identified, irrespective of actual HSCT.

Current DFS survival curves (estimating the proportion of patients disease-free over follow-up) were estimated using multistate models. Kaplan-Meier estimator was used for OS and cumulative incidences of transplantation were also estimated. Estimates are presented with $95 \%$ confidence intervals. Acute GVHD was analyzed as a binary outcome, with Firth's penalized logistic regression for adjusted analyses, to limit sparse data bias. A proportional cause-specific hazards model was used to compare the hazard of transplantation among groups, with Holm's correction for multiple testing. Gray's test was used to compare cumulative incidences. All tests were two-sided, at a 0.05 significance level. Analyses used the $\mathrm{R}$ statistical software version 3.6.0 R Core Team (2019). R: A language and environment for statistical computing. $\mathrm{R}$ Foundation for Statistical Computing, Vienna, Austria. https://www.R-project.org/. 


\section{Biology}

The molecular profiling of patients was performed by nextgeneration sequencing (NGS) using a capture-based custom NGS panel (Sophia Genetics) targeting relevant 23 myeloid genes. The sequencing was performed on a MiSeq instrument (Illumina). Bioinformatics was realized at Sophia Genetics (Switzerland) using the SOPHIA DDM software and significant variants were retained with a sensitivity of $1 \%$. The cytokine profile was analyzed with multiplex magnetic Luminex Assays (reference LXSAHM) from R\&D system. Luminex assays were performed according to the manufacturer's instructions and were read on a Bioplex MAGPIX Multiplex Reader from Biorad.

\section{Results}

\section{Patients}

Seventy-eight patients were included, but two patients were excluded from the follow-up and the analysis because of exclusion criteria violation. Patient characteristics are shown in Table 1. Median age was 59 years. Most patients were male $(66 \%)$, had primary MF $(70 \%)$, palpable splenomegaly (95\%), JAK2V617F mutation (68\%) and were at intermediate- 2 or high risk according to DIPSS (65\%). One patient died before the fourth-month post-inclusion and information about donor availability was available for the remaining 75 patients at 4 months. A donor was found in 64 patients: 18 HLA-matched sibling donor (MSD), 32 HLAmatched unrelated donor (UD10/10), and 14 HLA mismatched (UD9/10). Eleven patients had no donor and were followed in the arm "without a donor" (Fig. 1). Characteristics of the patients according to the donor availability at 4 months are shown in Table 1. Characteristics of the transplanted patients according to the donor are shown in Supplementary Table S1. In addition to the driver mutation, the most frequent additional mutations were in the ASXL1 (36\%), TET2 (25\%), EZH2 (18\%), SRSF2 (17\%), and U2AF1 (17\%) genes (Fig. 2). Median follow-up was 31 months.

\section{Response to ruxolitinib}

All patients initiated ruxolitinib $15 \mathrm{mg}$ BID at inclusion. Two patients stopped ruxolitinib within 3 months due to hematological toxicity (see SAE section). All other patients received ruxolitinib for at least 3 months and could be assessed for a 3-month response. Marrow biopsy was not performed at 3 months, due to the low probability of observing any difference compared with inclusion. At 3 months, 20 (26\%) patients had PR, 15 (20\%) patients had a spleen response and the remaining patients had no response (54\%). According to no donor, MSD, UD10/10, and UD9/10, PR was achieved in $3(25 \%), 6(33 \%), 11$ (34\%), and 0 patients. The median time from donor identification to transplantation ranges between 1 and 2.1 months and was not influenced by the response to ruxolitinib (data not shown). MF symptom assessment form (MFSAF) was assessed in 65 patients at 3 months and showed a $50 \%$ decrease in $17 \%$ of patients (Supplementary Fig. 2). Compared with the baseline, quality of life (QOLC30) questionnaires did not disclose marked changes on day 90 (Supplementary Fig. S2).

\section{Transplantation and splenectomy}

Among the 64 patients with a donor, 19 underwent a splenectomy before a scheduled transplantation $(6 / 18$ in MSD, 11/32 in UD10/10, 2/14 in UD9/10). SAE <21 days after splenectomy were reported in seven patients: six portal or splenic thrombosis and one acute cardiogenic shock. Two splenectomized patients did not undergo transplantation due to cardiac failure, both were scheduled for UD9/10 (Fig. 3). Fifty-nine (92\%) patients with a donor underwent HSCT. Although not meaningful on a clinical point of view, time from donor identification to HSCT was 14 days shorter in patients transplanted from MSD as compared to those transplanted from UD ( $p=0.036$ after correction for multiple testing). The probability of being transplanted was not different between MSD and unrelated donors (UD) (100\% vs. $89 \%$ ). Anti-thymoglobulin (ATG) was used in one SIB (6\%), 20 UD10/10 (67\%) and 11 UD9/10 (91\%). Of note, one patient who received an UD9/10 donor had no ATG but received post-transplant cyclophosphamide instead of pretransplant ATG. Details regarding transplant characteristics are available in Supplemental data. All patients came to transplantation on ruxolitinib which was stopped before conditioning regimen progressively in the first group of patients before amendment $(n=17)$ and abruptly after amendment $(n=42)$.

\section{Severe adverse events}

SAE are listed in Table 2 and grade 3-4 toxicities are available online in Supplementary Table S2. Heart failure, TLS, RWS occurred in seven, three, and three patients, respectively. Timing from inclusion, ruxolitinib withdrawal, and transplantation to these SAE are shown in Fig. 3. Briefly, cardiac failure occurred in variable conditions (pre or post-transplant, with or without ruxolitinib) and four patients had cardiogenic shock. TLS always occurred within $72 \mathrm{~h}$ from melphalan, all occurring before amendment for abrupt ruxolitinib discontinuation (so after progressive discontinuation). RWS have been suspected 
Table 1 Characteristics of all patients at inclusion.

\begin{tabular}{|c|c|c|c|c|c|}
\hline \multirow{2}{*}{ Characteristic } & \multicolumn{5}{|c|}{ Available donor at month four } \\
\hline & $\begin{array}{l}\text { All patients } \\
(N=76)\end{array}$ & $\begin{array}{l}\text { No donor } \\
\left(N=11^{\mathrm{a}}\right)\end{array}$ & $\begin{array}{l}\text { Sibling } \\
(N=18)\end{array}$ & $\begin{array}{l}\text { Unrelated } 10 / 10 \\
(N=32)\end{array}$ & $\begin{array}{l}\text { Unrelated } 9 / 10 \\
(N=14)\end{array}$ \\
\hline Median age (range)—year & $59(27-68)$ & $58(50-67)$ & $58(45-68)$ & $62(27-68)$ & $58(42-66)$ \\
\hline Female-no. $(\%)$ & $26(34)$ & $4(36)$ & $4(22)$ & $11(34)$ & $6(43)$ \\
\hline \multicolumn{6}{|l|}{ Myelofibrosis subtype-no. (\%) } \\
\hline Primary myelofibrosis & $53(70)$ & $8(73)$ & $12(67)$ & $20(62)$ & $12(86)$ \\
\hline Post-polycythemia vera myelofibrosis & $13(17)$ & $2(18)$ & $5(28)$ & $6(19)$ & $0(0)$ \\
\hline Post-essential thrombocythemia myelofibrosis & $10(13)$ & $1(9)$ & $1(6)$ & $6(19)$ & $2(14)$ \\
\hline Median time since diagnosis (range)—months & $4(0-229)$ & $4(1-196)$ & $24(2-77)$ & $3(0-72)$ & $5(0-229)$ \\
\hline No. missing & 1 & 1 & 0 & 0 & 0 \\
\hline \multicolumn{6}{|l|}{ Prior therapies } \\
\hline Hydroxyurea-no. $(\%)$ & $32(42)$ & $3(27)$ & $7(39)$ & $15(47)$ & $7(50)$ \\
\hline Pegylated interferon alfa-2a-no. (\%) & $6(8)$ & $1(9)$ & $3(17)$ & $2(6)$ & $0(0)$ \\
\hline Radiotherapy-no. (\%) & $1(1)$ & $0(0)$ & $0(0)$ & $0(0)$ & $1(7)$ \\
\hline \multicolumn{6}{|l|}{ JAK2 status-no. (\%) } \\
\hline V617F & $52(68)$ & $9(82)$ & $11(61)$ & $23(72)$ & $9(64)$ \\
\hline CALR & $17(22)$ & $2(18)$ & $4(22)$ & $6(19)$ & $4(29)$ \\
\hline MPL & $1(1)$ & $0(0)$ & $1(6)$ & $0(0)$ & $0(0)$ \\
\hline None & $6(8)$ & $0(0)$ & $2(11)$ & $3(9)$ & $1(7)$ \\
\hline ASXL1 mutation一no. (\%) & $27(36)$ & $3(27)$ & $6(33)$ & $13(41)$ & $5(36)$ \\
\hline \multicolumn{6}{|l|}{ Karyotype-no. (\%) } \\
\hline Normal & $33(43)$ & $4(36)$ & $8(44)$ & $15(47)$ & $5(36)$ \\
\hline Abnormal & $33(43)$ & $6(55)$ & $10(56)$ & $10(31)$ & $7(50)$ \\
\hline No mitosis & $2(3)$ & $0(0)$ & $0(0)$ & $2(6)$ & $0(0)$ \\
\hline Unknown & $8(11)$ & $1(9)$ & $0(0)$ & $5(16)$ & $2(14)$ \\
\hline Cytogenetic high risk-no. (\%) & $8(12)$ & $3(30)$ & $1(6)$ & $3(12)$ & $1(8)$ \\
\hline No. missing & 9 & 1 & 0 & 7 & 1 \\
\hline Previous transfusions-no. (\%) & $33(43)$ & $4(36)$ & $6(33)$ & $15(47)$ & $7(50)$ \\
\hline \multicolumn{6}{|l|}{ Hematologic parameters } \\
\hline Median hemoglobin (range)—g/dL & $9.8(5.7-15.8)$ & $9.4(7.2-15.8)$ & $11.4(7.9-14.2)$ & $9.8(6.8-15.1)$ & $9.7(7.7-13.4)$ \\
\hline Median platelet count (range) $-10^{9} / \mathrm{L}$ & $190(29-1130)$ & $155(88-379)$ & $200(58-468)$ & $174(29-839)$ & $202(61-1130)$ \\
\hline Median absolute neutrophil count (range) $-10^{9} / \mathrm{L}$ & $5.9(0.5-50.6)$ & $5.5(2.4-14.8)$ & $9.7(1.0-50.6)$ & $4.9(0.5-34.4)$ & $7.6(1.0-36.0)$ \\
\hline No. missing & 1 & 0 & 0 & 0 & 1 \\
\hline Median white-cell count (range) $-10^{9} / \mathrm{L}$ & $9(1-65)$ & $8(4-22)$ & $13(1-65)$ & $8(2-61)$ & $9(2-57)$ \\
\hline Median peripheral-blood blast count (range)-percent & $0.0(0.0-9.0)$ & $1.0(0.0-5.0)$ & $1.0(0.0-6.0)$ & $0.0(0.0-9.0)$ & $0.0(0.0-7.0)$ \\
\hline Palpable spleen—no. (\%) & $72(95)$ & $10(91)$ & $16(89)$ & $32(100)$ & $13(93)$ \\
\hline Median spleen length below costal margin (range) $-\mathrm{cm}$ & $10(0-28)$ & $7(2-14)$ & $13(3-26)$ & $10(0-25)$ & $11(2-28)$ \\
\hline No. missing & 3 & 0 & 0 & 2 & 1 \\
\hline Constitutional symptoms-no. (\%) & $43(57)$ & $8(73)$ & $12(67)$ & $12(38)$ & $11(79)$ \\
\hline Median MSAF score (range)—units & $24(7-72)$ & $35(9-59)$ & $22(8-63)$ & $21(7-72)$ & $26(9-61)$ \\
\hline No. missing & 4 & 2 & 1 & 0 & 1 \\
\hline \multicolumn{6}{|l|}{ Age-adjusted DIPSS—no. (1\%) } \\
\hline Intermediate-1 & $27(36)$ & $2(18)$ & $6(33)$ & $14(44)$ & $4(29)$ \\
\hline Intermediate- 2 & $31(41)$ & 7 (64) & $6(33)$ & $12(38)$ & $6(43)$ \\
\hline High & $18(24)$ & $2(18)$ & $6(33)$ & $6(19)$ & $4(29)$ \\
\hline
\end{tabular}

${ }^{\mathrm{a}} \mathrm{A}$ patient died before month four and was not considered in the description according to donor availability at month four. 
Fig. 1 Flow chart of the JAK ALLO protocol. Number of patients enrolled in the trial, number of patients who were available for evaluation for ruxolitinib response, and number of patients who have a donor and could receive transplantation.

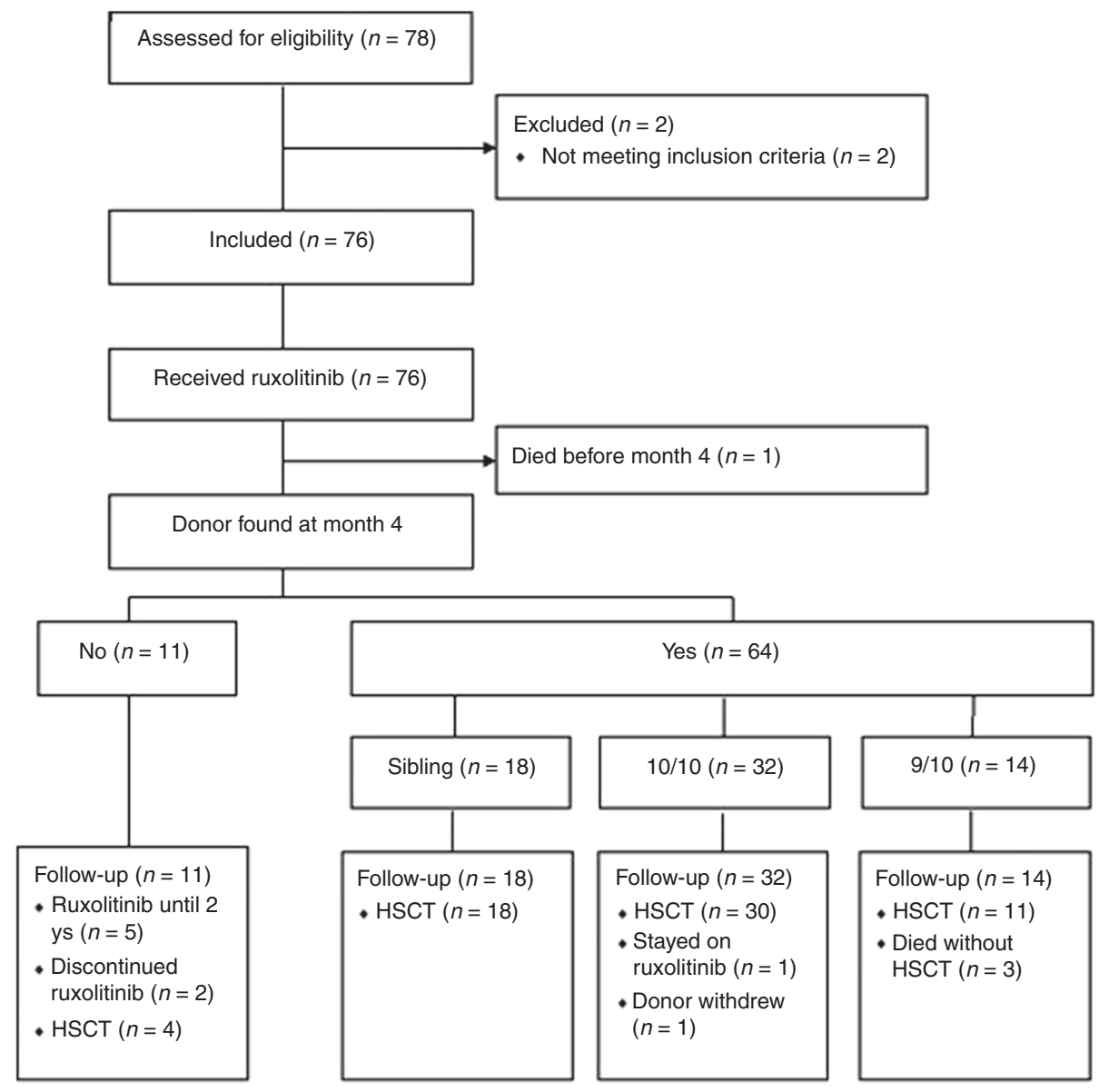

Patients

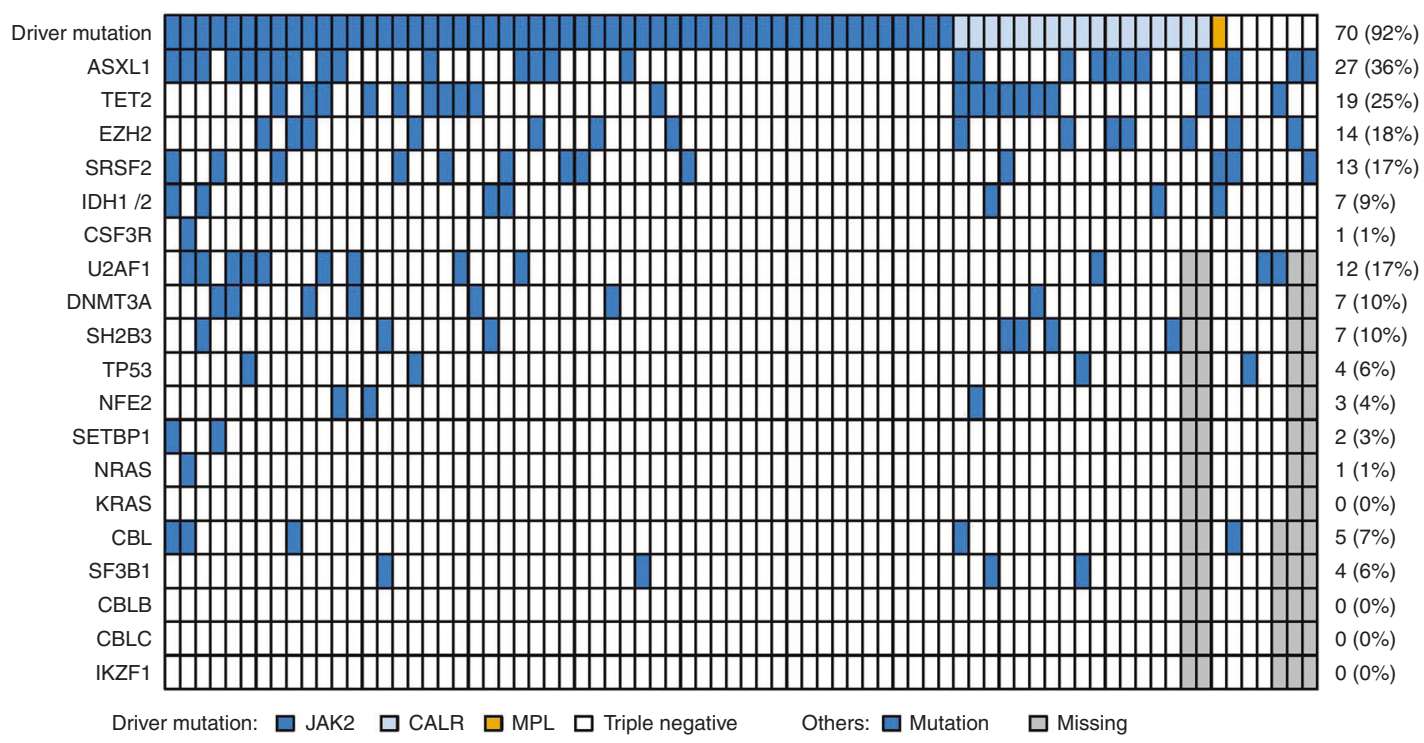

Fig. 2 Somatic mutations in patients at inclusion. The molecular profiling of patients was performed by next-generation sequencing (NGS) using a capture-based custom NGS panel (Sophia Genetics) targeting relevant 23 myeloid genes. (ASXL1; CALR; CBL; CBLB; CBLC; CSF3R; DNMT3A; EZH2; IDH1; IDH2; IKZF1; JAK2; KRAS; MPL; NFE2; NRAS; SETBP1; SF3B1; SH2B3; SRSF2;
TET2; TP53; U2AF1). Libraries were prepared on $200 \mathrm{ng}$ extracted from whole blood DNA (Qiagen) and the sequencing was performed on a MiSeq instrument (Illumina). Bioinformatics were realized at Sophia Genetics (Switzerland) using the SOPHIA DDM software and significant variants were retained with a sensitivity of $1 \%$. The first line shows the driver mutations (JAK2, MPL, CALR). 
but only one patient has symptoms that were reverted by ruxolitinib re-instauration. Clinical details are provided in the legend of Fig. 3.

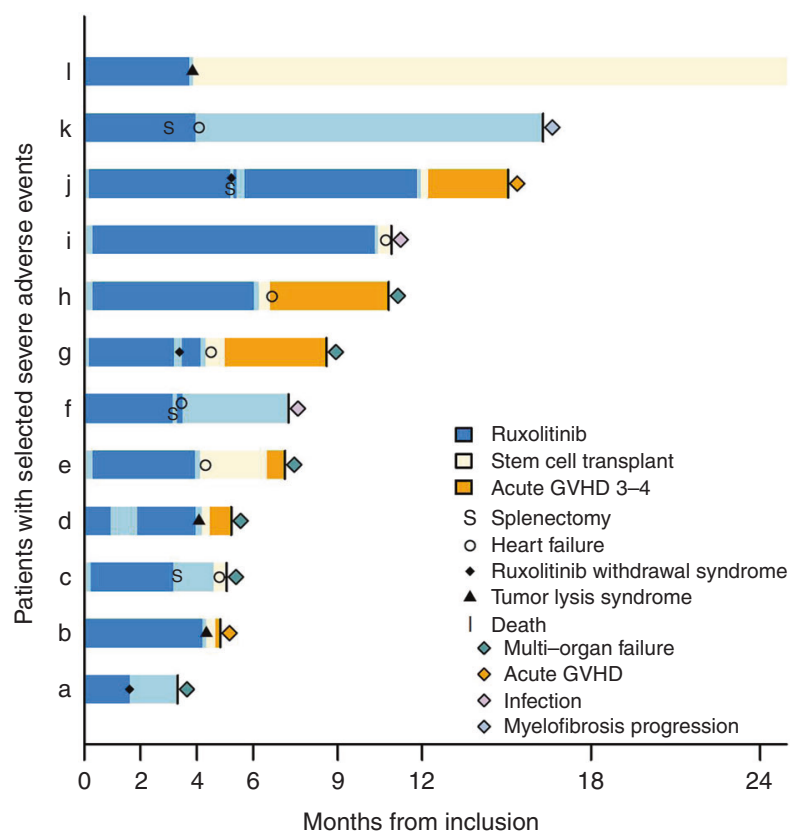

Fig. 3 Monitoring of patients with heart failure, ruxolitinib withdrawal syndrome, or tumor lysis syndrome. Each line is one patient identified by a letter from "a" to "i". Deep blue bars represent the period of ruxolitinib treatment, pale blue bars represent the period off ruxolitinib, pale yellow is the period after transplantation, without grade 3-4 acute GVHD, while orange bars are the period after transplantation, with grade 3-4 acute GVHD. Ruxolitinib withdrawal syndrome, tumor lysis syndrome, and heart failure are reported with specific icons. Causes of death are reported with a colored diamond. The clinical presentation was heterogeneous: four patients had a acute cardiogenic shock, requiring immediate intensive care for ventilation and amine vasopressor (patients "c", "e", "f", "k"), while three other patients had heart failure managed in standard care unit (patients "g", "h", "I"). Among the four suffering from cardiogenic shock, one occurred on ruxolitinib after a transient ruxolitinib discontinuation 5 days before (during splenectomy), one occurred during conditioning regimen with anti-thymoglobulin and the two others in the early post-transplant phase. Cardiogenic shocks occurred during systemic inflammatory response syndrome (SIRS) which was not microbiologically documented. All but one patients with cardiogenic shock had a complete cardiac recovery without ruxolitinib reintroduction. The three other patients with cardiac SAE had moderate left ventricular ejection dysfunction, associated with major fluid retention in two patients and respiratory distress for one patient. Three patients developed a possible RWS without any cardiac failure (patients "a", "g", "j"). Patient "a" had ruxolitinib discontinuation for pancytopenia grade 4 and splenomegaly worsening under treatment. He developed a febrile renal failure, followed by multi-organ failure and death, with blood cultures positive for Enterococcus faecium. Patient " $\mathrm{g}$ " developed fever, increasing splenomegaly, and hypotension after ruxolitinib was transiently stopped, which resolved after re-initiation of ruxolitinib. Patient " $\mathrm{j}$ " developed fever during the peri-operative period of the splenectomy, which resolved under steroids, but also had a splenic thrombosis and attack of gout, which may also explain the inflammatory process (as well as surgery). Three patients had TLS (patient "b", "d", "l") 24 to $72 \mathrm{~h}$ after Melphalan and one of them had significant renal failure reaching the stage of clinical TLS in the Cairo-Bishop definitions [41].

\section{Engraftment and GVHD}

Among the patients who received HSCT, all had successful engraftment at 4 months and one patient had late rejection. Cumulative incidence of grade $2-4$ hyperacute GVHD was $32 \%(21-44)$ for all patients, $22 \%(7-44)$ after MSD, 33\% (17-50) after UD10/10, and 46\% (15-72) after UD9/10. Cumulative incidence of grade $2-4$ acute GVHD on day 100 was $66 \%(52-77)$ for all patients, $61 \%$ (34-80) after MSD, 63\% (43-78) after U10/10D, and 82\% (37-96) after UD9/10. Cumulative incidence of grade $3-4$ acute GVHD was $44 \%$ (31-56) on day 100 for all patients, 28\% (10-49) in MSD, 40\% (22-57) in UD10/10, and 82\% (37-96) in UD9/10. Cumulative incidence of chronic GVHD was $33 \%$ (21-45) at 12 months and 37\% (24-60) at 24 months. The cumulative incidence of chronic GVHD was higher for MSD (75\% at 24 months, vs. 23\% for U10/10D and $11 \%$ for UD9/10, $p=0.0003$ ). Patients who were splenectomized before HSCT had less frequently grade 2-4 acute GVHD (53\%, vs $71 \%$ ) but it did not reach significance when adjusted with type of donor (SIB, UD10/ 10, UD9/10), ATG, and DIPSS (HR: 0.46, 95\%CI: $0.12-1.71$ ). Similarly, abrupt vs. progressive ruxolitinib discontinuation had no influence on GVHD occurrence (HR: 0.73, 95\%CI: 0.18-2.89) after adjustment with same covariates (Table 3).

\section{Survival}

OS in all patients was 68\% (95\% CI: 59-80) and 55\% (95\% CI: 44-67) at 12 and 24 months. In transplanted patients, 2year OS was $59 \%$ with splenectomy and $49 \%$ without splenectomy and splenectomy was not significant significantly associated with mortality (Supplementary Table S3), 2-year OS was 35\% if progressive ruxolitinib discontinuation vs. $59 \%$ if abrupt discontinuation, the hazard ratio after adjustment was 0.48 (95\%CI: 0.21-1.08) (Table 3). The type of donor was associated with mortality while there was a trend to higher mortality in older patients and higher DIPSS (Table 3 and Fig. 4a). Somatic mutations had no impact on outcome (Supplementary Table S4). Among transplanted patients, DFS was 52\% (39-64) and $46 \%$ (33-58) at 12 and 24 months after transplantation. According to the type of donor, DFS at 12 and 24 months was $83 \%(60-94)$ and $77 \%$ (53-90), 40\% (23-56) and 36\% (19-52), 34\% (11-59) and 23\% (5-47) with a MSD, UD10/ 10 or UD9/10, respectively (Fig. 4b). NRM in transplanted patients was $42 \%$ at 12 months and $46 \%$ at 24 months. NRM was higher after unrelated transplant (23\% with MSD vs. $50 \%$ after U10/10D and $77 \%$ after UD9/10, $p=0.014$ ). In the Cox model, abrupt ruxolitinib discontinuation was associated with lower risk of NRM without reaching significance (Table 3). 
Table 2 List of severe adverse events (SAEs).

\begin{tabular}{|c|c|c|c|}
\hline Severe adverse event (SAE) & $\begin{array}{l}\text { No. } \\
\text { patients }(\%)\end{array}$ & $\begin{array}{l}\text { No. events } \\
\text { before } \\
\text { HSCT }^{\text {a }}\end{array}$ & $\begin{array}{l}\text { No. } \\
\text { events } \\
\text { after } \\
\text { HSCT }^{\text {b }}\end{array}$ \\
\hline Infection & $19(25)$ & 8 & 19 \\
\hline Heart failure & $7(9)$ & 2 & 5 \\
\hline Portal vein thrombosis & $6(8)$ & 6 & 0 \\
\hline Renal failure & $3(4)$ & 0 & 3 \\
\hline $\begin{array}{l}\text { Ruxolitinib withdrawal } \\
\text { syndrome (RWS) }\end{array}$ & $3(4)$ & 3 & 0 \\
\hline $\begin{array}{l}\text { Tumor lysis } \\
\text { syndrome (TLS) }\end{array}$ & $3(4)$ & 3 & 0 \\
\hline Anemia & $2(3)$ & 2 & 0 \\
\hline Pancreatitis & $2(3)$ & 1 & 1 \\
\hline Severe anemia & $2(3)$ & 2 & 0 \\
\hline $\begin{array}{l}\text { Acute respiratory distress } \\
\text { syndrome (ARDS) }\end{array}$ & $1(1)$ & 1 & 0 \\
\hline Cancer & $1(1)$ & 1 & 0 \\
\hline Cystic lymphangioma & $1(1)$ & 1 & 0 \\
\hline Depression & $1(1)$ & 0 & 1 \\
\hline Diarrhea & $1(1)$ & 0 & 1 \\
\hline Encephalopathy & $1(1)$ & 0 & 1 \\
\hline Engraftment syndrome & $1(1)$ & 0 & 1 \\
\hline Graft rejection & $1(1)$ & 0 & 1 \\
\hline Intestinal ischemia & $1(1)$ & 1 & 0 \\
\hline Meningoencephalitis & $1(1)$ & 0 & 1 \\
\hline Osteonecrosis & $1(1)$ & 1 & 0 \\
\hline $\begin{array}{l}\text { Post-transplant } \\
\text { lymphoproliferative } \\
\text { disease (PTLD) }\end{array}$ & $1(1)$ & 0 & 1 \\
\hline $\begin{array}{l}\text { Systemic inflammatory } \\
\text { response syndrome (SIRS) }\end{array}$ & $1(1)$ & 1 & 0 \\
\hline $\begin{array}{l}\text { Sinusoidal obstruction } \\
\text { syndrome (SOS) }\end{array}$ & $1(1)$ & 0 & 1 \\
\hline Splenic infarction & $1(1)$ & 1 & 0 \\
\hline Thrombocytosis & $1(1)$ & 1 & 0 \\
\hline Transfusion reaction & $1(1)$ & 1 & 0 \\
\hline VIIth nerve palsy & $1(1)$ & 1 & 0 \\
\hline $\begin{array}{l}\text { Worsening of a } \\
\text { previous cancer }\end{array}$ & $1(1)$ & 0 & 1 \\
\hline
\end{tabular}

Adverse Events (AEs) were graded according to the Common Terminology Criteria for Adverse Events (CTCAE) version 4.03 http://evs.nci.nih.gov/ftp1/CTCAE.

${ }^{\mathrm{a}}$ All patients.

${ }^{\mathrm{b}}$ Transplanted patients.

\section{Cytokine analyses}

Most cytokines were downregulated by ruxolitinib, except four out of 26 cytokines (CCL11, Trappin-2, MMP3, EPO) (Fig. 5). A specific profile could be determined in patients who responded to ruxolitinib, compared with patients who did not respond. Similarly, a specific profile was associated with patients who developed GVHD, compared with patients who did not. Some similarities were observed in non-responders to ruxolitinib and patients who developed GVHD, such as downregulation of CXCL10, trappin-2, REG3alpha, CD40L, and upregulation of EPO, CCL2, and IL8, but the profiles were not totally superimposable (Fig. 5 and Supplementary Fig. S3).

\section{Discussion}

The JAK ALLO study was conducted hypothesizing that short course of ruxolitinib given prior to the transplantation may be beneficial for the post-transplant outcome in patients with myelofibrosis. In this trial, ruxolitinib was stopped before the conditioning regimen, either progressively (first part of the trial) or abruptly (after amendment). The probability to undergo the transplantation after ruxolitinib initiation was excellent (92\%). This trial is one of the only prospective trial considering myelofibrosis patients at time of transplantation indication (and not just before transplantation) allowing the analysis of potential events between ruxolitinib initiation and transplantation. While outcome was very good with MSD, it was disappointing with UD. Higher mortality in patients who received a transplant from an unrelated donor was due to higher rate of hyperacute and grades 3-4 GVHD. In particular, more than $80 \%$ of patients transplanted from an HLA mismatched unrelated donor developed grade 3-4 acute GVHD. However, poor survival in patients with UD9/10 donor also included 2 patients who were never transplanted due to cardiac failure; all patients who were transplanted from UD9/10 had no response to ruxolitinib eventually explaining worse outcome in these patients who had possibly an advanced disease which is a potential bias in the study. Splenectomy was still performed in $30 \%$ of patients while it was $37 \%$ of patients in the last French registry study, showing that it was not dramatically decreased by ruxolitinib use [6]. One can argue that the ruxolitinib time was too short to achieve a significant spleen reduction, this point could not be assessed in the trial. Regarding the relative good survival in patients without a donor, the trial was not designed to analyze this secondary objective and more patients would be necessary to confirm this observation.

Mortality and acute GVHD risk have been regularly reported as higher in myelofibrosis patients receiving a transplant from an unrelated donor [7, 23, 24]. A combination of factors might explain this higher mortality: the use of melphalan, reported as more toxic than busulfan; [25] the absence of systematical use of anti-thymoglobulin (73\% in unrelated transplant); an inflammatory cytokine profile; the combination of cyclosporine and mycophenolate GVHD 
Table 3 Multivariable analysis for outcomes after transplantation.
Fig. 4 Survival and diseasefree survival. a. Overall survival (OS) was estimated from 4 months according to donor search (no donor, HLAmatched sibling donor, matched unrelated donor, mismatched unrelated donor). One patient was excluded because he died before donor search result. With HLA-matched sibling donor as a reference, Hazard ratio was 4.1 (1.4-12.2) with matched unrelated donor, 7.8 (2.4-25.5) with mismatched unrelated donor and 2.9 (0.82-10.3) without a donor, overall $\mathrm{p}$ value is 0.002 . b. Disease-free survival (DFS) was estimated in patients alive at 4 months and is shown according to the type of donor. Patients without a donor are not represented, because none of them reached a complete remission.

\begin{tabular}{|c|c|c|c|c|c|c|}
\hline & \multirow[b]{2}{*}{$N(\%)$} & \multicolumn{2}{|c|}{ NRM } & \multirow{2}{*}{$\begin{array}{l}\text { Acute GVHD } \\
N(\%)\end{array}$} & \multicolumn{2}{|c|}{ OS } \\
\hline & & $N$ & $\mathrm{CIF}^{\mathrm{a}}(95 \%$ CI) @ 24 months. & & $N$ & OS (95\% CI)@ 24 months \\
\hline Progressive & $17(29 \%)$ & 11 & $65 \%(36-83)$ & $12(71 \%)$ & 13 & $35 \%(19-67)$ \\
\hline Abrupt & $42(71 \%)$ & 16 & $39 \%(24-53)$ & $27(64 \%)$ & 17 & $59 \%(45-76)$ \\
\hline & & & Adjusted HR $(95 \% \mathrm{CI})$ & $\begin{array}{l}\text { Adjusted OR (95\% } \\
\text { CI) }\end{array}$ & & Adjusted HR $(95 \% \mathrm{CI})$ \\
\hline Abrupt stopping & & & $0.50(0.21-1.18)$ & $0.77(0.20-2.93)$ & & $0.48(0.21-1.08)$ \\
\hline $10 / 10 v$ SIB & & & $4.65(1.47-14.7)$ & $2.11(0.41-10.9)$ & & $5.35(1.72-16.6)$ \\
\hline $9 / 10 v \mathrm{SIB}$ & & & $5.93(1.74-20.2)$ & $4.94(0.55-44.7)$ & & $6.28(1.84-21.4)$ \\
\hline Age (per year) & & & $1.02(0.97-1.08)$ & $1.02(0.95-1.09)$ & & $1.03(0.97-1.09)$ \\
\hline DIPSS Int- $2 v$ Int- 1 & & & $0.96(0.36-2.55)$ & $1.43(0.39-5.27)$ & & $0.98(0.38-2.50)$ \\
\hline DIPSS High $v$ Int- 1 & & & $2.39(0.85-6.70)$ & $2.57(0.56-11.9)$ & & $2.24(0.83-6.05)$ \\
\hline ATG & & & - & $0.44(0.09-2.09)$ & & - \\
\hline
\end{tabular}

$C I F$ cumulative incidence, $H R$ Hazard ratio, $O R$ odd ratio.

Bold values indicate statistical significance $p<0.05$.

a

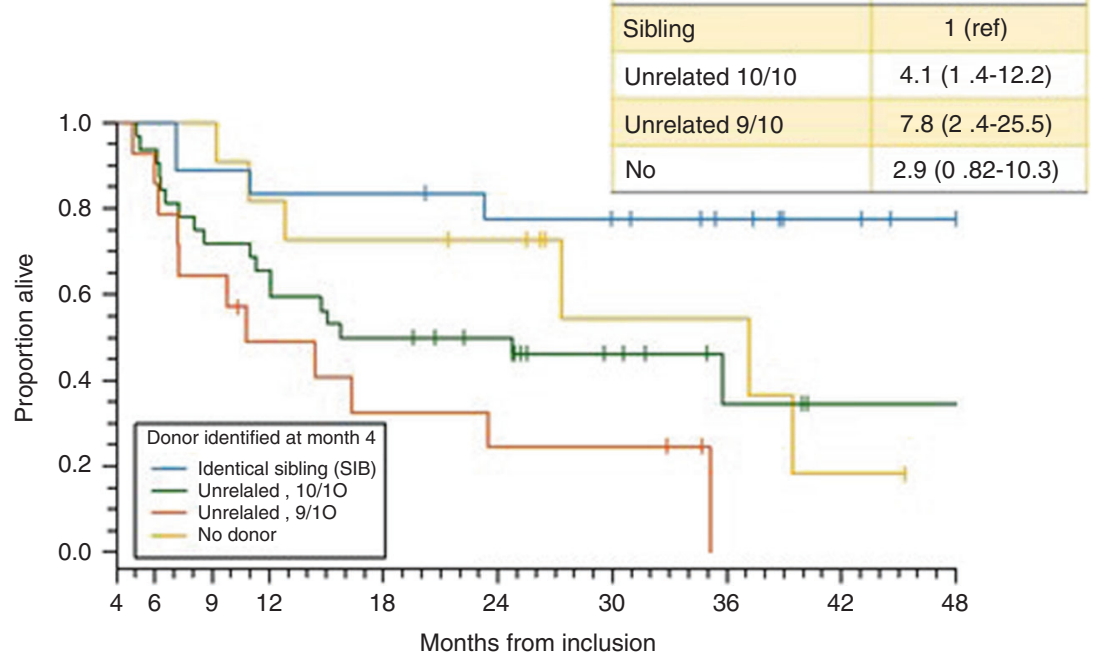

No. at risk

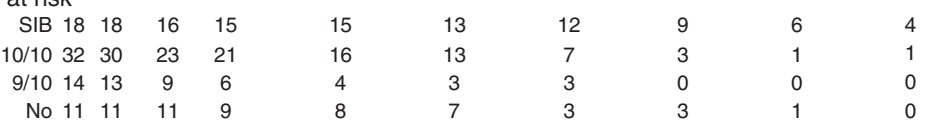

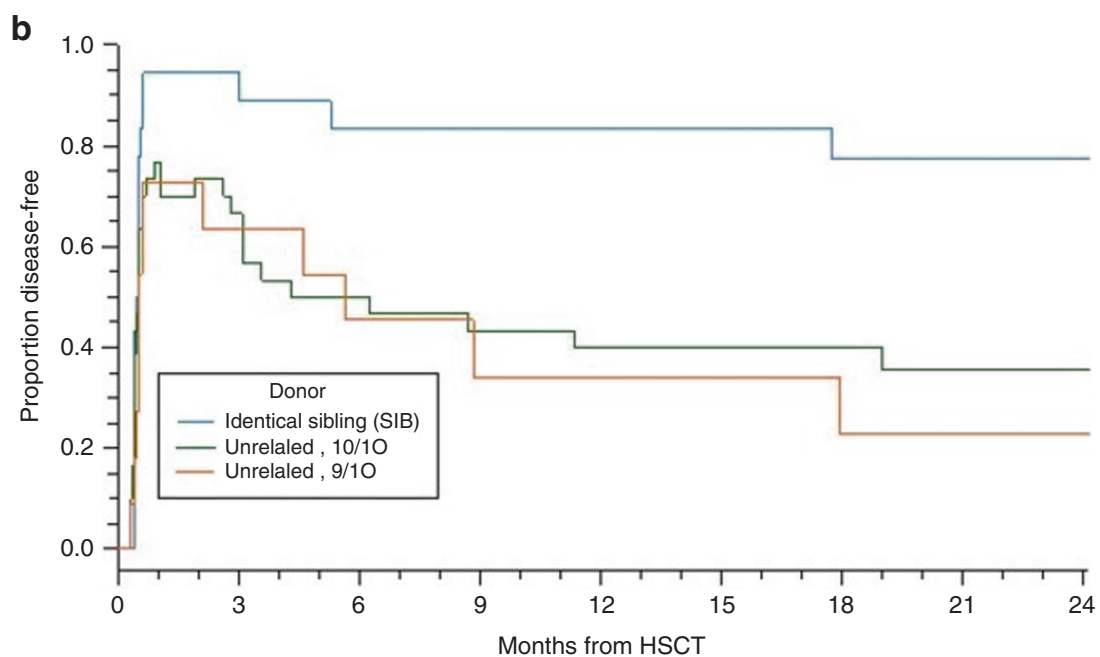


a

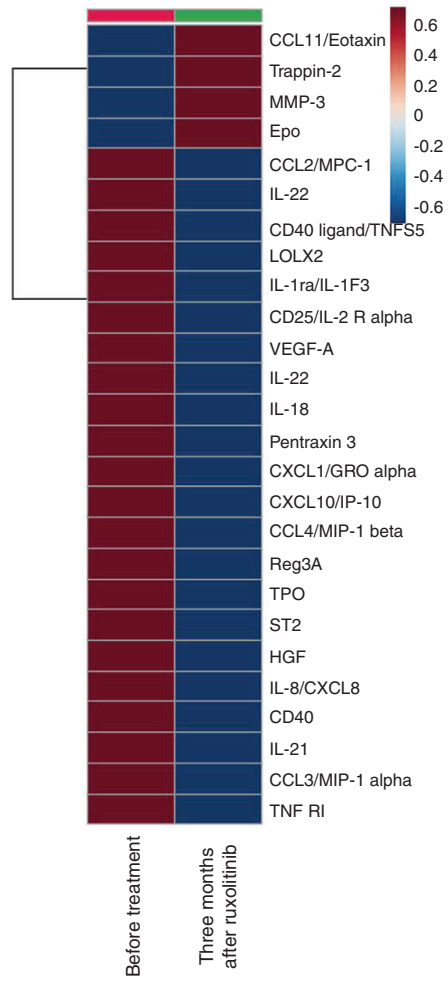

b

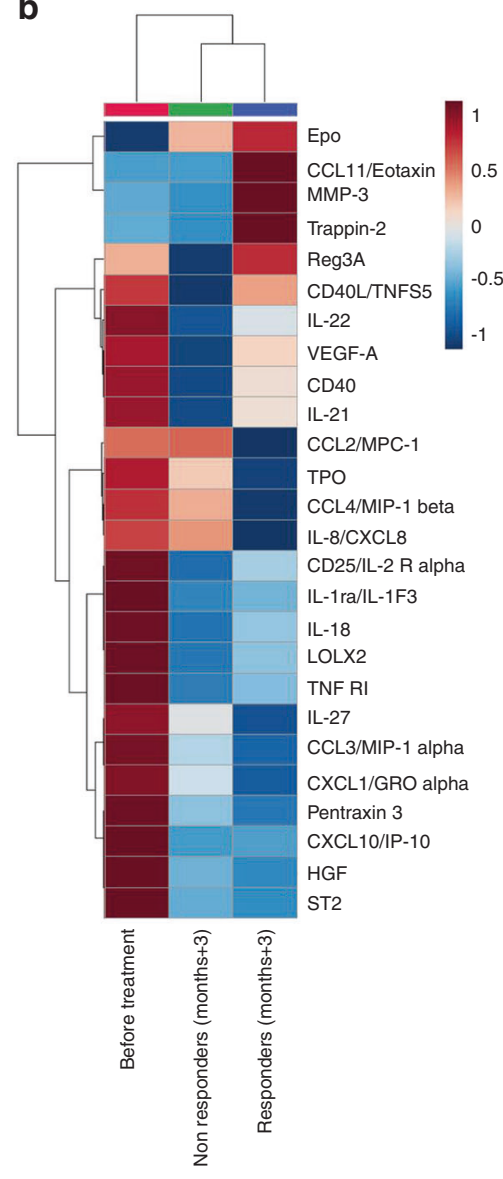

C

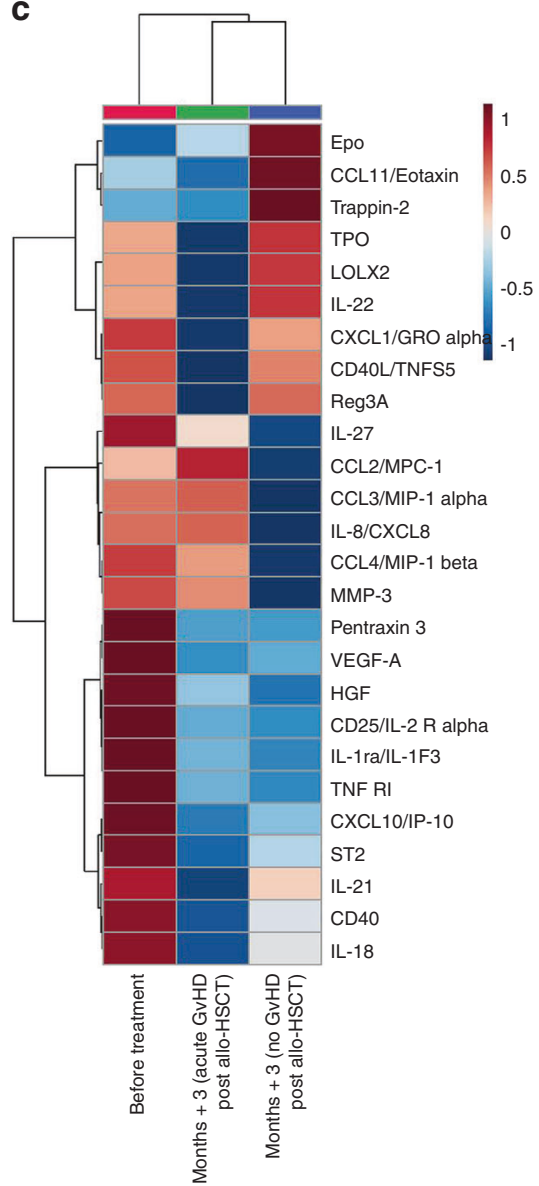

Fig. 5 Variation of cytokine expression. 46 cytokines were measured:B7-H1/PD-L1， CCL2/MPC-1， CD40 LIGAND/TNFS5, CXCL10/IP-10, FGF-BASIC, GM-CSF, IL-10, IL-15, IL-1BETA/IL1F2, IL-2, IL-4, IL-6, TNF-ALPHA, CCL3/MIP-1 ALPHA, CCL11/ Eotaxin, CCL4/MIP-1 beta, CXCL1/GRO alpha, IL-8/CXCL8, GCSF, IFN-alpha, IL-12 p70, IL-17A, IL-1ra/IL-1F3, IL-33, IL-5, IL-7, TRAIL, CD40, HGF, IL-21, IL-27, MMP-3, Pentraxin 3, ST2, TPO, LOLX2, Epo, IL-18, IL-22, CD25/IL-2 R alpha, VEGF-A, Reg3A, TNF RI, Trappin-2, IL-9, OSM. Serum levels of selected biomarkers are shown as heat maps (Panels a, b, c) they were selected by partial least square discriminant analysis for biomarkers with variable importance in the projection (VIP) score $>1$ ). Each row constitutes the mean for several patients. Each column constitutes the period of serum collection. Blue and red denote markers that are expressed at lower and higher levels, respectively. a Cytokine profile from inclusion to 3 -

prophylaxis and the use of peripheral-blood stem cells for all patients. ATG was associated with a lower risk of acute GVHD in our trial without reaching significance but statistical power was limited due to the low number of patients $(H R<0.5)$. Several prospective phase 3 studies have recently reported lower risk of GVHD with ATG (in different diseases) confirming that ATG may be recommended in these situations [26-28]. More recently, the question of ATG has been also addressed in the setting of HLAmatched sibling donor in a phase 3 trial and in an EBMT registry for myelofibrosis patients, showing a decreased risk month post-inclusion. This panel shows the increase or decrease of biomarkers value (logarithmic transformation of the means and autoscaling) in biomarkers obtained from patients at inclusion $(n=54$, before ruxolitinib) compared with 3 months after inclusion $(n=55$, on ruxolitinib). The a shows that cytokines are down-expressed, except for the first four cytokines (first to fourth row). b Cytokines profile in responders and non-responders to ruxolitinib. This panel shows the cytokine value at inclusion and at month three in responders $(n=13)$ and non-responders $(n=42)$ to ruxolitinib. Comparing column two to column three, we can see which cytokines are differentially expressed in responders and non-responders. c Cytokine profile in patients with or without GVHD. This panel shows cytokine values at inclusion and at month three in patients who will develop GVHD and in patients who will not.

of acute or chronic GVHD [29, 30]. Regarding GVHD prophylaxis, it has been reported by CIBMTR in AML patients received RIC and MUD that cyclosporine and mycophenolate were followed by higher risk of acute GVHD as compared to the combination cyclosporine and methotrexate [31]. The role of ruxolitinib on post-transplant outcome is difficult to determine, since the trial was not designed to compare patients with and without ruxolitinib. We suspected that progressive ruxolitinib discontinuation before conditioning regimen was associated with higher risk of complication and mortality. We could observe that the 
risks of acute GVHD, NRM, and overall mortality were reduced when ruxolitinib was abruptly stopped but it did not reach significance, again, possibly due to low number of patients. We could observe that the cytokine profile was modified by ruxolitinib, as previously reported by others [32]. In addition, non-responders to ruxolitinib, and patients who developed acute GVHD, had close cytokines profiles, suggesting that an inappropriate biological response to ruxolitinib could be associated with the subsequent development of acute GVHD.

The rebound of cytokines associated with ruxolitinib withdrawal has been suspected in this trial, as well as by others $[20,21,33,34]$, but it was extremely difficult to prove. Firstly, there is no worldwide accepted definition of the syndrome, so we had to give a definition in order to prospectively capture and declare potential RWS. Furthermore, there were multiple interactions, which could explain symptoms presented by the patients. TLS which was not included in the definition of RWS, occurred in 5\% of transplanted patients and always in days after melphalan.

Gupta et al. reported 21 patients who were transplanted after ruxolitinib treatment abruptly stopped before a reduced-intensity conditioning regimen with busulfan and fludarabine. There was no specific syndrome that could be attributed to RWS, but a poor accrual and a significant number of graft failures led to the study being prematurely closed [35]. Of note, acute GVHD was frequently reported (64\%), as well as chronic GVHD (76\%). Finally, outcome on an intention-to-treat basis was good, with 2-year OS at $61 \%$ for the MSD, and $70 \%$ for the UD arm. Salit et al. reported 28 patients from a prospective study treated by ruxolitinib and who received HSCT [36]. These patients mostly received a myeloablative conditioning regimen, and ruxolitinib was stopped during the conditioning regimen. They also reported a high incidence of grade II-IV acute GVHD (78\%). No RWS was reported. A retrospective assessment by Kroger et al. explored the question of ruxolitinib given prior and after transplantation in 11 patients with myelofibrosis (MF) and observed a very low incidence of acute GVHD [37]. In another retrospective study, serious adverse events of rebound splenomegaly necessitating splenectomy and respiratory distress syndrome were reported in two patients, delaying HSCT [38].

Finally, the use of ruxolitinib, which has immunosuppressive properties, has not increased the risk of disease progression and did not seem to compromise graft-versusmyelofibrosis effect, as relapse rate was minimal in our study.

To conclude, short-term ruxolitinib therapy before transplantation is associated with a high probability to be transplanted in patients with a donor. The results were poorer in patients transplanted from unrelated donor than in those transplanted from SIB. Many different reasons may have contributed to this difference. In view of these results, the question of the timing of transplantation in newly diagnosed patients can be raised, especially in patients without a MSD, taking also into account that a delayed transplantation may expose to the risk of AML transformation, a stage with a very poor outcome after transplantation $[39,40]$. The future national trials will test other conditions that may decrease GVHD and mortality in myelofibrosis patients transplanted from an unrelated donor.

Acknowledgements We thank all centers, investigators, data managers, and patients participating to JAK ALLO, we thank Novartis for its financial support; we thank l'Agence de Biomédecine (ABM) for supporting the biological study.

Author contributions MR, BD, GS, and JJK designed the paper. MR, $\mathrm{CO}, \mathrm{JOB}, \mathrm{FB}, \mathrm{AH}, \mathrm{AC}, \mathrm{ED}, \mathrm{SC}, \mathrm{CB}$, IYA, MD, DM, PT, LL, FB, FS, LV, SNG, JC, AV, BD, GS, JJK contributed to patient recruitment. All co-authors performed research. RP made the statistical analysis. MHS made cytokine experiments. BC made molecular analyses. MR, GS, RP, and JJK wrote the paper. All authors approved the paper. VR and NR did the data management. GS and JJK contributed equally.

\section{Compliance with ethical standards}

Conflict of interest This trial has received financial support from Novartis. BD: Advisory board Novartis; EF: Advisory board Novartis; DM: Speaker bureau for Novartis and Incyte; GS: Speaker bureau Novartis, Incyte, Elsalys, Consultant Alexion; IYA: honorarium and advisory board Novartis; JJK: consultant for Novartis, Celgene, and AOP Orphan; received research funding from Novartis. MD: research funding by Novartis and Jazzpharma MR: research funding by Novartis, Neovii; speaker bureau for Novartis and Incyte

Publisher's note Springer Nature remains neutral with regard to jurisdictional claims in published maps and institutional affiliations.

\section{References}

1. Grinfeld J, Nangalia J, Baxter EJ, Wedge DC, Angelopoulos N, Cantrill R, et al. Classification and personalized prognosis in myeloproliferative neoplasms. N Engl J Med. 2018;379:1416-30.

2. Kröger NM, Deeg JH, Olavarria E, Niederwieser D, Bacigalupo $\mathrm{A}$, Barbui $\mathrm{T}$, et al. Indication and management of allogeneic stem cell transplantation in primary myelofibrosis: a consensus process by an EBMT/ELN international working group. Leukemia. 2015;29:2126-33.

3. Deeg HJ, Gooley TA, Flowers MED, Sale GE, Slattery JT, Anasetti C, et al. Allogeneic hematopoietic stem cell transplantation for myelofibrosis. Blood. 2003;102:3912-8.

4. Kerbauy DMB, Gooley TA, Sale GE, Flowers MED, Doney KC, Georges GE, et al. Hematopoietic cell transplantation as curative therapy for idiopathic myelofibrosis, advanced polycythemia vera, and essential thrombocythemia. Biol Blood Marrow Transpl. 2007; 13:355-65.

5. Patriarca F, Bacigalupo A, Sperotto A, Isola M, Soldano F, Bruno $\mathrm{B}$, et al. Allogeneic hematopoietic stem cell transplantation in myelofibrosis: the 20-year experience of the Gruppo Italiano Trapianto di Midollo Osseo (GITMO). Haematologica. 2008;93:1514-22. 
6. Robin M, Tabrizi R, Mohty M, Furst S, Michallet M, Bay J-O, et al. Allogeneic haematopoietic stem cell transplantation for myelofibrosis: a report of the Société Française de Greffe de Moelle et de Thérapie Cellulaire (SFGM-TC). Br J Haematol. 2011;152:331-9.

7. Kröger N, Holler E, Kobbe G, Bornhäuser M, Schwerdtfeger R, Baurmann $\mathrm{H}$, et al. Allogeneic stem cell transplantation after reduced-intensity conditioning in patients with myelofibrosis: a prospective, multicenter study of the Chronic Leukemia Working Party of the European Group for Blood and Marrow Transplantation. Blood. 2009;114:5264-70.

8. Ballen KK, Shrestha S, Sobocinski KA, Zhang M-J, Bashey A, Bolwell BJ, et al. Outcome of transplantation for myelofibrosis. Biol Blood Marrow Transpl. 2010;16:358-67.

9. Rondelli D, Goldberg JD, Isola L, Price LS, Shore TB, Boyer M, et al. MPD-RC 101 prospective study of reduced-intensity allogeneic hematopoietic stem cell transplantation in patients with myelofibrosis. Blood. 2014;124:1183-91.

10. Gupta V, Malone AK, Hari PN, Ahn KW, Hu Z-H, Gale RP, et al. Reduced-intensity hematopoietic cell transplantation for patients with primary myelofibrosis: a cohort analysis from the center for international blood and marrow transplant research. Biol Blood Marrow Transpl. 2014;20:89-97.

11. Samuelson Bannow BT, Salit RB, Storer BE, Stevens EA, Wu D, Yeung $\mathrm{C}$, et al. Hematopoietic cell transplantation for myelofibrosis: the dynamic international prognostic scoring system plus risk predicts post-transplant outcomes. Biol Blood Marrow Transpl. 2018;24:386-92.

12. Cervantes F, Pereira A. Does ruxolitinib prolong the survival of patients with myelofibrosis? Blood. 2017;129:832-7.

13. Verstovsek S, Mesa RA, Gotlib J, Gupta V, DiPersio JF, Catalano $\mathrm{JV}$, et al. Long-term treatment with ruxolitinib for patients with myelofibrosis: 5-year update from the randomized, double-blind, placebo-controlled, phase 3 COMFORT-I trial. J Hematol Oncol. 2017;10:55.

14. Dupriez B, Morel P, Demory JL, Lai JL, Simon M, Plantier I, et al. Prognostic factors in agnogenic myeloid metaplasia: a report on 195 cases with a new scoring system. Blood. 1996;88:1013-8.

15. Passamonti F, Cervantes F, Vannucchi AM, Morra E, Rumi E, Cazzola M, et al. Dynamic international prognostic scoring system (DIPSS) predicts progression to acute myeloid leukemia in primary myelofibrosis. Blood. 2010;116:2857-8.

16. Tefferi A, Barosi G, Mesa RA, Cervantes F, Deeg HJ, Reilly JT, et al. International Working Group (IWG) consensus criteria for treatment response in myelofibrosis with myeloid metaplasia, for the IWG for Myelofibrosis Research and Treatment (IWG-MRT). Blood. 2006;108:1497-503.

17. Przepiorka D, Weisdorf D, Martin P, Klingemann HG, Beatty P, Hows J, et al. Consensus conference on acute GVHD grading. Bone Marrow Transpl. 1994;15:825-8.

18. Shulman HM, Kleiner D, Lee SJ, Morton T, Pavletic SZ, Farmer E, et al. Histopathologic diagnosis of chronic graft-versus-host disease: National Institutes of Health Consensus Development Project on Criteria for Clinical Trials in Chronic Graft-versus-Host Disease: II. Pathology Working Group Report. Biol Blood Marrow Transpl. 2006;12:31-47.

19. Saliba RM, de Lima M, Giralt S, Andersson B, Khouri IF, Hosing C, et al. Hyperacute GVHD: risk factors, outcomes, and clinical implications. Blood. 2007;109:2751-8.

20. Tefferi A, Pardanani A. Serious adverse events during ruxolitinib treatment discontinuation in patients with myelofibrosis. Mayo Clin Proc. 2011;86:1188-91.

21. Dai T, Friedman EW, Barta SK. Ruxolitinib withdrawal syndrome leading to tumor lysis. J Clin Oncol. 2013;31:e430-32.

22. A'Hern RP. Sample size tables for exact single-stage phase II designs. Stat Med. 2001;20:859-66.
23. McLornan D, Szydlo R, Koster L, Chalandon Y, Robin M, Wolschke C, et al. Myeloablative and reduced-intensity conditioned allogeneic hematopoietic stem cell transplantation in myelofibrosis: a retrospective study by the chronic malignancies working party of the european society for blood and marrow transplantation. Biol Blood Marrow Transpl. 2019;25:2167-71.

24. Hernández-Boluda JC, Pereira A, Kröger N, Beelen D, Robin M, Bornhäuser $\mathrm{M}$, et al. Determinants of survival in myelofibrosis patients undergoing allogeneic hematopoietic cell transplantation. Leukemia. 2021;35:215-24.

25. Robin M, Porcher R, Wolschke C, Sicre de Fontbrune F, Alchalby $\mathrm{H}$, Christopeit M, et al. Outcome after transplantation according to reduced-intensity conditioning regimen in patients undergoing transplantation for myelofibrosis. Biol Blood Marrow Transpl. 2016;22:1206-11.

26. Walker I, Panzarella T, Couban S, Couture F, Devins G, Elemary $\mathrm{M}$, et al. Pretreatment with anti-thymocyte globulin versus no antithymocyte globulin in patients with haematological malignancies undergoing haemopoietic cell transplantation from unrelated donors: a randomised, controlled, open-label, phase 3, multicentre trial. Lancet Oncol. 2016;17:164-73.

27. Soiffer RJ, Kim HT, McGuirk J, Horwitz ME, Johnston L, Patnaik $\mathrm{MM}$, et al. Prospective, randomized, double-blind, phase III clinical trial of Anti-T-lymphocyte globulin to assess impact on chronic graft-versus-host disease-free survival in patients undergoing HLA-matched unrelated myeloablative hematopoietic cell transplantation. J Am Soc Clin Oncol. 2017;35:4003-11.

28. Finke J, Bethge WA, Schmoor C, Ottinger HD, Stelljes M, Zander AR, et al. Standard graft-versus-host disease prophylaxis with or without anti-T-cell globulin in haematopoietic cell transplantation from matched unrelated donors: a randomised, open-label, multicentre phase 3 trial. Lancet Oncol. 2009;10:855-64.

29. Kröger N, Solano C, Wolschke C, Bandini G, Patriarca F, Pini M, et al. Antilymphocyte globulin for prevention of chronic graftversus-host disease. N. Engl J Med. 2016;374:43-53.

30. Robin M, Chevret S, Koster L, Wolschke C, Yakoub-Agha I, Bourhis $\mathrm{JH}$, et al. Antilymphocyte globulin for matched sibling donor transplantation in patients with myelofibrosis. Haematologica. 2019;104:1230-6.

31. Chhabra S, Liu Y, Hemmer MT, Costa L, Pidala JA, Couriel DR, et al. Comparative analysis of calcineurin inhibitor-based methotrexate and mycophenolate mofetil-containing regimens for prevention of graft-versus-host disease after reduced-intensity conditioning allogeneic transplantation. Biol Blood Marrow Transpl. 2019;25:73-85.

32. Verstovsek S, Kantarjian H, Mesa RA, Pardanani AD, CortesFranco J, Thomas DA, et al. Safety and efficacy of INCB018424, a JAK1 and JAK2 inhibitor, in myelofibrosis. N. Engl J Med. 2010;363:1117-27.

33. Coltro G, Mannelli F, Guglielmelli P, Pacilli A, Bosi A, Vannucchi AM. A life-threatening ruxolitinib discontinuation syndrome. Am J Hematol. 2017;92:833-8.

34. Beauverd Y, Samii K. Acute respiratory distress syndrome in a patient with primary myelofibrosis after ruxolitinib treatment discontinuation. Int J Hematol. 2014;100:498-501.

35. Gupta V, Kosiorek HE, Mead A, Klisovic RB, Galvin JP, Berenzon $\mathrm{D}$, et al. Ruxolitinib therapy followed by reducedintensity conditioning for hematopoietic cell transplantation for myelofibrosis: myeloproliferative disorders research consortium 114 study. Biol Blood Marrow Transpl. 2019;25:256-64.

36. Salit RB, Scott BL, Stevens EA, Baker KK, Gooley TA, Deeg HJ. Pre-hematopoietic cell transplant Ruxolitinib in patients with primary and secondary myelofibrosis. Bone Marrow Transpl. 2020;55:70-6.

37. Kröger N, Shahnaz Syed Abd Kadir S, Zabelina T, Badbaran A, Christopeit M, Ayuk F, et al. Peritransplantation ruxolitinib prevents acute graft-versus-host disease in patients with myelofibrosis 
undergoing allogenic stem cell transplantation. Biol Blood Marrow Transpl. 2018;24:2152-6.

38. Shanavas M, Popat U, Michaelis LC, Fauble V, McLornan D, Klisovic R, et al. Outcomes of allogeneic hematopoietic cell transplantation in patients with myelofibrosis with prior exposure to janus kinase 1/2 inhibitors. Biol Blood Marrow Transpl. 2016;22:432-40.

39. Kröger N, Eikema D-J, Köster L, Beelen D, de Wreede LC, Finke $\mathrm{J}$, et al. Impact of primary disease on outcome after allogeneic stem cell transplantation for transformed secondary acute leukaemia. Br J Haematol. 2019;185:725-32.

40. Ruggiu M, Cassinat B, Kiladjian J-J, Raffoux E, Giraudier S, Robin M, et al. Should transplantation still be considered for Ph1negative myeloproliferative neoplasms in transformation? Biol Blood Marrow Transplant. 2020;26:1160-70.

41. Cairo MS, Bishop M. Tumour lysis syndrome: new therapeutic strategies and classification. Br J Haematol. 2004;127:3-11.

\section{Affiliations}

Marie Robin $\mathbb{D}^{1} \cdot$ Raphael Porcher $\mathbb{D}^{2,3} \cdot$ Corentin Orvain $^{4} \cdot$ Jacques-Olivier Bay $^{5} \cdot$ Fiorenza Barraco ${ }^{6} \cdot$ Anne Huynh $^{7}$. Amandine Charbonnier ${ }^{8} \cdot$ Edouard Forcade $\left.^{9}\right)^{9} \cdot$ Sylvain Chantepie ${ }^{10} \cdot$ Claude Bulabois $^{11}$. Ibrahim Yakoub-Agha $\mathbb{1}^{12} \cdot$ Marie Detrait ${ }^{13}$ - David Michonneau ${ }^{14}{ }^{14} \cdot$ Pascal Turlure $^{15} \cdot$ Nicole Raus $^{16}$. Françoise Boyer ${ }^{4} \cdot$ Felipe Suarez ${ }^{17} \cdot$ Laure Vincent $^{18}{ }^{18}$. Stéphanie N. Guyen ${ }^{19} \cdot$ Jérôme Cornillon ${ }^{20}$. Alban Villate $^{21} \cdot$ Brigitte Dupriez $^{22} \cdot$ Bruno Cassinat $^{23} \cdot$ Valérie Rolland $^{24} \cdot$ Marie Hélène Schlageter $^{23}$. ${\text { Gérard Socié }{ }^{10}{ }^{14} \cdot \text { Jean-Jacques Kiladjian }}^{25}$

1 Hôpital Saint-Louis, APHP, Service d'hématologie greffe, Paris, France

2 Université de Paris, Epidemiology and Statistics Research Center (CRESS), Institut National de la Santé et de la Recherche Médicale (INSERM), Institut National de la Recherche Agronomique (INRA), Paris, France

3 Centre d'Épidémiologie Clinique, Assistance Publique-Hôpitaux de Paris (AP-HP), Hôpital Hôtel-Dieu, Paris, France

4 CHU Angers, Angers, France

5 CHU Clermont-Ferrand, Clermont-Ferrand, France

6 CHU Lyon Sud, Lyon, France

7 CHU Toulouse, Toulouse, France

8 CHU Amiens, Amiens, France

9 CHU Bordeaux, service d'Hématologie et Thérapie Cellulaire, Bordeaux, France

10 CHU Caen, Caen, France

11 CHU Grenoble Alpes, Grenoble, France

12 CHU de Lille, Univ Lille, INSERM U1286, Infinite, Lille, France
13 CHRU de Nancy, Institut Louis Mathieu, Université de Lorraine, Vandoeuvre-lès-Nancy, France

14 Hôpital Saint-Louis, APHP, service d'hématologie greffe, U976Université de Paris, Paris, France

15 CHU Limoges, Limoges, France

16 Hôpital Lyon Sud, SFGM-TC, Lyon, France

17 Hôpital Necker, CHU, APHP, Paris, France

18 Hôpital Saint-Eloi, CHU Montpellier, Montpellier, France

19 Hôpital Pitié Salpétrière, APHP, Paris, France

20 Institut de Cancérologie de la Loire, Saint-Etienne, France

21 Hôpital Bretonneau CHRU de Tours, Tours, France

22 Centre hospitalier. Route de La Bassée., Lens, France

23 Hôpital Saint-Louis, APHP, laboratoire de biologie cellulaire, Paris, France

24 CHU Lyon Sud, FILO, Lyon, France

25 Hôpital Saint-Louis, APHP, Centre d'investigation clinique, Paris, France 